SUBJECT AREAS:

PLANT MOLECULAR

BIOLOGY

PLANT DEVELOPMENT

Received

6 December 2013

Accepted

16 January 2014

Published

4 February 2014

Correspondence and requests for materials should be addressed to

P.K.T. (prabodht@

hotmail.com;

prabodh†@nbri.res.in)

\title{
Expression of OsMATE 1 and OsMATE2 alters development, stress responses and pathogen susceptibility in Arabidopsis
}

\author{
Manish Tiwari' ${ }^{1}$ Deepika Sharma' ${ }^{1}$, Munna Singh², Rudra Deo Tripathi' \& Prabodh Kumar Trivedi'
}

\begin{abstract}
'National Botanical Research Institute, Council of Scientific and Industrial Research (CSIR), Rana Pratap Marg, Lucknow-226 001, INDIA, ${ }^{2}$ Department of Botany, University of Lucknow, Lucknow-226007, INDIA
\end{abstract}

Multidrug and Toxic compound Extrusion proteins (MATE) are a group of secondary active transporters with ubiquitous occurrences in all domains of life. This is a newly characterized transporter family with limited functional knowledge in plants. In this study, we functionally characterised two members of rice MATE gene family, OSMATE1 and OSMATE2 through expression in heterologous system, Arabidopsis. Expression of OsMATEs in Arabidopsis altered growth and morphology of transgenic plants. Genome-wide expression analysis revealed modulation of genes involved in plant growth, development and biotic stress in transgenic lines. Transgenic plants displayed sensitivity for biotic and abiotic stresses. Elevated pathogen susceptibility of transgenic lines was correlated with reduced expressions of defence related genes. Promoter and cellular localization studies suggest that both MATEs express in developing and reproductive organs and are plasma-membrane localised. Our results reveal that OSMATE1 and OsMATE2 regulate plant growth and development as well as negatively affect disease resistance.

M ultidrug and Toxic compound Extrusion proteins (MATE) are a family of secondary active transporters which utilize electrochemical gradient of membrane maintained by ATPases for their transport activity ${ }^{1}$. Members of this transporter family have ubiquitous occurrence in all the organisms including human and plants. The first member of this family (NorM) was characterized from Vibriopara haemolyticus, which effluxes nofloxacin and ciprofloxacin outside of the cells in an energy dependent way ${ }^{2}$. Most of the earlier studies suggest that MATE proteins act as an efflux pump that export majority of drugs and xenobiotic compounds outside the cell, and largely contribute to the drug resistance in bacteria. Recent reports indicate that MATE proteins function in antiport manner and drive the substrate transport in exchange with $\mathrm{H}^{+} / \mathrm{Na}^{+}$depending on the organism. Studies suggest that $\mathrm{H}^{+}$coupling is operational in plants, which is substituted by $\mathrm{Na}^{+}$in bacteria. Though structure and mechanism of action of MATEs have not been studied in detail, recent investigations revealed conformation and transport behaviour of MATE proteins ${ }^{3,4}$. Conversely to designated name "multidrug", numerous studies revealed that MATE proteins have stringent substrate specificity and facilitate the movement of specific compounds ${ }^{5}$. Presence of homologous putative MATEs has been identified from several organisms through comparative genomics. A genome wide scanning and analysis revealed that 58 MATE paralogues are present in Arabidopsis genome ${ }^{6}$. In general, MATE proteins from various source organisms have a conserved domain and share at least $40 \%$ amino acid sequence homology ${ }^{3}$.

In higher plants, studies demonstrated that MATE proteins are mainly involved in the transport and trafficking of xenobiotic and small organic molecules ${ }^{7}$. The foremost characterized Arabidopsis MATE protein, AtDTX1, was demonstrated to export norfloxacin due to its ability to restore drug tolerance in the norfloxacin sensitive bacterial mutant (kam3 mutant) ${ }^{8}$. Recently, evidences for role of MATEs in the plants are rapidly accumulating. Depending on the established functions of this gene family in plants, MATEs have been grouped into three major classes. The first group of the MATE has been demonstrated to be associated with disease resistance in Arabidopsis. Several Arabidopsis mutants have been identified and examined for understanding the molecular mechanism of pathogen resistance. Among them, enhanced disease susceptibility mutant (eds5) of Arabidopsis displayed a reduced basal resistance during pathogen interaction. The fine genetic mapping of the eds 5 locus identified that $E D S 5$, a member of the MATE transporter family, was localised within this locus, and substantially contributed to disease tolerance ${ }^{9}$. The relation of $E D S 5$ with disease tolerance has been explored very recently and demonstrated that EDS5 export salicylic acid (SA) outside chloroplast, where SA synthesis takes place ${ }^{10,11}$. Similarly, ADS1 (activated disease susceptability1), a negative regulator for the disease resistance, was another 
MATE identified in Arabidopsis showing involvement during pathogen infection ${ }^{12}$. A second group of MATE proteins is known for exporting small organic molecules such as citrate outside the cell which acts as a ligand molecule to bind aluminium (Al) in the rhizosphere. Unlike to other metals, Al toxicity is much pronounced in acidic soil and several genetic studies were performed to map the locus responsible for $\mathrm{Al}$ tolerance in plants. A locus for $\mathrm{Al}$ tolerance, $A t_{S B}$, was identified that encode a MATE via positional cloning in sorghum. This SbMATE has been shown to efflux the citrate from cell and forming non-toxic complexes with $\mathrm{Al}$ in soil solution ${ }^{13}$. Similar to sorghum, MATE proteins from Hordeum ${ }^{14}$, Arabidopsis ${ }^{15}$, Triticum $^{16}$ and maize ${ }^{17}$ have been shown to participate in Al tolerance. Tandem duplication of MATE paralogues in the maize genome have been demonstrated to offer another level of transcriptional regulation to provide adaptation of maize to $\mathrm{Al}$ toxicity ${ }^{18}$.

Another, third group of MATE transporters are involved in trafficking of secondary metabolites in vacuoles. Usually, vacuoles are the major repository site of most of the conjugated form of flavonoids comprising mainly flavonols, anthocyanins and flavone glycoside. The transport and storage of these flavonoides into vacuole are mediated by the different class of transporters on tonoplast ${ }^{19}$. A member of MATE gene family (TT12) was elucidated for sequestration of proanthocyanidins in vacuoles of seed that leads to pigmentation of seed coat $^{20}$. The kinetic study of vesicles isolated from TT12 expressing yeast revealed that it can specifically transport glycosidic form like epicatechin 3'-O-glucoside and cyanidin 3-O glucoside $^{5}$. Consistent to the TT12, MATEs from Medicago and Vitis have been characterized for mobilization of flavonoids in cell organelles ${ }^{21-23}$. Similar to the role in vacuolar flavonoids sequestration, MATE proteins are also recognised for alkaloids trafficking into vacuoles in tobacco ${ }^{24,25}$.

Apart from these functions FRD3, a MATE citrate efflux transporter, was shown to involved in Fe transport towards stele region and hence assisted in distribution of Fe throughout aerial parts in Arabidopsi ${ }^{26}$. Curiously, one member of MATE gene family (Mdt1) was shown to regulate the glucose assimilation, and which is thought to be necessary for nutrient utilization, sporulation and pathogenicity in fungi ${ }^{27}$. In an another report, role of a MATE-like transporter $(Z R Z)$ has been demonstrated in organ initiation as overexpression of this MATE led to diverse morphological changes in Arabidopsis ${ }^{28}$. Therefore, it seems that MATEs govern the diverse physiological functions in the plants which need to be elucidated. Likewise to Arabidopsis, genome-wide analysis of MATEs encoding loci in rice revealed the presence of large members of this gene family however, function of only a few members has been elucidated as yet ${ }^{29,30}$. Our earlier study suggested that expression of some of the genes encoding MATEs is up-regulated in rice seedlings exposed to arsenic (As) stress $^{31}$. Of these loci, we have investigated the role of two gene, OsMATE1 (Os03g08900) and OsMATE2 (Os05g48040) using heterologous system, Arabidopsis, in this study. Our observations suggest that these MATEs play crucial role in plant growth, development and in stress responses.

\section{Results}

In silico identification and analysis of rice MATEs. In order to identify MATE genes present in the rice genome, BLASTP analysis at Rice Genome Annotation Project using Arabidopsis MATEs as query sequences was performed. A total of 53 members of the rice MATE family were identified (Supplementary Table S2). To study the evolutionary relationship between identified rice MATE proteins, a maximum likelihood phylogenetic tree was constructed using all the MATE proteins from Arabidopsis as well as from other plants with well established functions. The analysis suggests that rice MATE proteins clustered in different groups of Arabidopsis and other known MATEs from different plants.
As very limited information about the function of rice MATE proteins is available, we have selected two arsenic responsive members $^{31}$, OsMATE1 (Os03g08900) and OsMATE2 (Os05g48040), for functional studies. Though both of MATEs show same responses with As, whereas grouped in two distinct clades (Supplementary Fig. S1). These rice MATEs, OsMATE1 and OsMATE2 encode polypeptide comprising the 489 and 500 amino acid residues respectively and shares $36 \%$ identities and $53 \%$ similarity with each other within $90 \%$ query cover. Furthermore, trans-membrane helix prediction through TMHMM indicated the presence of 12 trans-membrane helices in both MATEs (Supplementary Fig. S2). This preliminary analysis suggests that OsMATE1 and OsMATE2 were membrane bound proteins and possibly engage in transport activity across membrane.

Expression of OsMATE1 and OsMATE2 alter growth in Arabidopsis. In order to investigate the function of OSMATE1 and OsMATE2, full-length cDNAs were expressed in Arabidopsis under control of a CaMV35S promoter. The presence and expression of transgene was confirmed through genomic and semiquantitative RTPCR (Supplementary Fig. S3). Depending upon relative expression and phenotypic appearance, three independent lines expressing OsMATE1 and OsMATE2 were selected for further study. Up to two weeks of germination in soil, growth pattern of the all transgenic lines was similar to WT plants. However, after two weeks of growth, transgenic lines expressing OsMATE1 or OsMATE2 displayed a distinguishable growth pattern in comparison to WT plants, but very similar to each other. The visible changes at the stage of three week old plants among all transgenic lines were quite prevalent (Supplementary Fig. S3, Fig. 1) that consist petiole length, leaf size and rosette leaf arrangement in vegetative parts.

The pattern of rosette leaves of the mature plants was different in comparison to WT plants without any change in the number of leaves (Fig. 1a). Petiole length and rosette leaf size of the transgenic lines were significantly increased compared to the WT (Fig. 1b, c). Collectively, the expression of OsMATE1 and OsMATE2 resulted variation in vegetative tissues, including the leaf size and altered pattern of rosette development. To authenticate these inferences, growth of two T-DNA insertional mutants of Arabidopsis MATEs (SALK_045655C and SALK_124549C) having close sequence similarity with OSMATE1 and OSMATE2 were analysed. Interestingly, a cognate growth pattern of salk lines with WT plants was observed (Supplementary Fig. S4a). Altogether, these observations suggest that OSMATE1 and OSMATE2 play important role in the growth and development of plants.

Altered floral morphology in transgenic lines expressing OsMATE1 and OsMATE2. Aside to morphological changes, variations in flowering time and flower size were observed in all the transgenic lines expressing OsMATE1 and OsMATE2 in comparison to WT plants (Fig. 2). An early flowering was observed in transgenic lines under normal growth conditions. Flowering time in transgenic lines was significantly reduced (up to four days) relative to WT plants (Fig. 2) but rosette leaf numbers at bolting stage were identical in transgenic lines and WT plants. Flowering time of salk mutants and WT plants were found similar and no significant difference was observed during initiation of bolting (Supplementary Fig. S4b). In addition to the alteration in flowering time, significantly reduced size of mature flower (length and width) was observed in transgenic lines compared to WT (Fig. 3). Though silique size of each transgenic line was comparable to WT, the number of siliques per plant were significantly higher (up to 50\%) in comparison to WT (Fig. 4b). Transgenic lines also produced smaller seeds as compared to WT, although the viability of seeds was not compromised (Fig. 4b). Similar to these differences of reproductive organs, seed number produced per silique in all the transgenic lines was significantly higher corresponding to WT plants (Fig. 4c). Taken together, these 
B
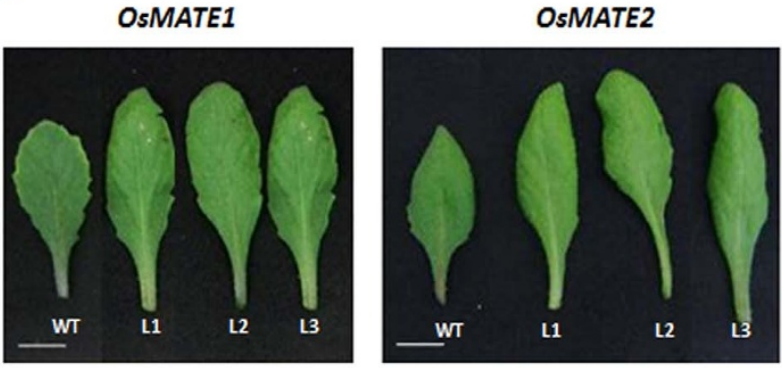

$\mathrm{C}$

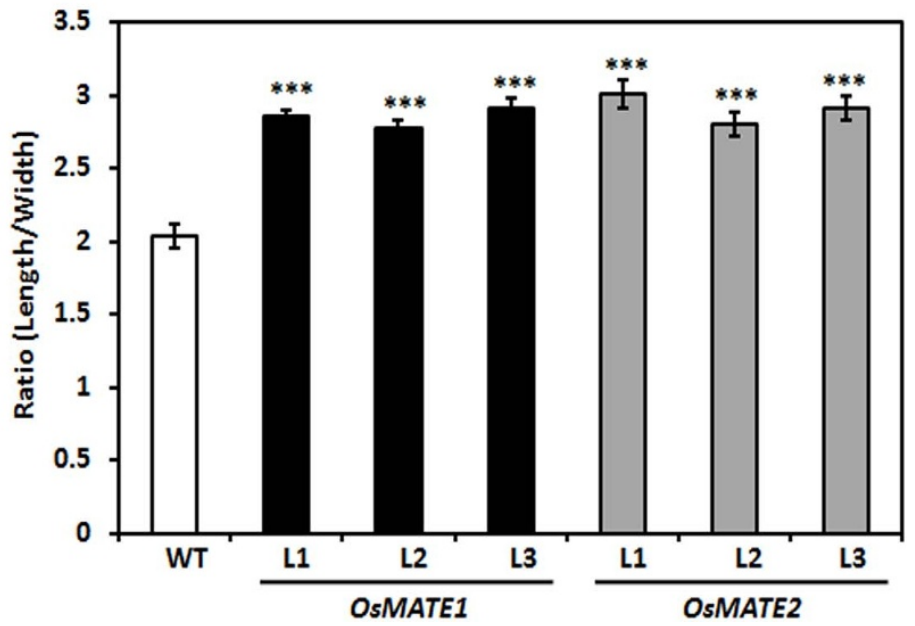

Figure $1 \mid$ Alteration of leaf morphology in transgenic lines. (A) Leaf sizes and pattern of each rosette leaf beginning from the oldest one of WT and one line representing OSMATE1 and OsMATE2 transgenic plants. Plants were grown for 4 week under $16 \mathrm{~h}$ light-8 h dark photoperiod before photograph. (B) Photograph showing the difference in leaf size ( $9^{\text {th }}$ leaf of rosette from oldest one) of WT and 3 representative lines of OsMATE1 and OsMATE2 transgenic plant. White line indicates the scale bar $(1 \mathrm{~cm})$. (C) Ratio of length and width of leaf $\left(9^{\text {th }}\right.$ position $)$ of transgenic lines and WT. Bars are mean and error bars represent \pm SE of 8-10 individual plants. Level of significance was evaluated by One-way ANOVA (Newman-Keuls used as post hoc test). *** Significantly different from WT $(\mathrm{P}<0.0001)$.

results suggest that expression of OsMATE1 or OsMATE2 in Arabidopsis develops many phenotypic changes which include reduced time of transition from juvenile to flowering stage, morphology of flowers, silique number, seed size and silique number produced per plants. However, such a macroscopic changes were not observed in salk lines.

Genome wide expression analysis in the transgenic lines. To address reason for phenotypic changes in transgenic plants, modulation of transcriptome was analyzed using Affymetrix ATH1 (22K) array of three week old WT and transgenic line expressing OsMATE1. At this stage, clear phenotypic changes between WT and transgenic lines were visible. After normalization of cel files through d-Chip, a significantly modulated expression ( $1.6 \geq$ fold, p values 0.05 ) of 39 genes in transgenic lines compared to WT plants were observed. Of these, expression of 14 and 25 genes were up- and down-regulated, respectively in the transgenic line (Table 1 and 2). The majority of genes up-regulated were related to DNA binding proteins and transcription factors controlling the diverse metabolic and physiological processes in plants. Out of most notable modulated expression, pentatricopeptide (PPR) repeat-containing protein upregulated nearly 3 fold (Table 1 ). Characterized PPR proteins in Arabidopsis are known to be localized in mitochondria and participate in the post transcriptional modification of RNA. The impaired or loss of function of PPR lead to reduced NADH dehydrogenase activity and consequently a compromise defence response in Arabidopsis ${ }^{32,33}$. Enhanced expression of RD20 (Responsive to Desiccation 20) and CAX7 (calcium exchanger 7) was also seen, which are involved in salinity stress. A significant up-regulation of NAC6 and NAC32 was observed in transgenic plants. NAC domain containing proteins are highly studied transcription factor family in plants and are known to regulate growth and development of plants and drought, desiccation, salinity and ABA response $\mathrm{e}^{34}$. Interestingly, expression of a Jumonji transcription factor (jmjC domain-containing protein) was enhanced in transgenic lines. In Arabidopsis, jmjC domain-containing protein (Jmj30) is known as the key determinant of circadian rhythm and regulates the flowering time in a cooperative manner with central oscillator, circadian clock associated 1 and late elongated hypocotyl ${ }^{35}$. Expression of all of the up-regulated genes was further validated through qRT-PCR (Fig. 5) that followed the same pattern of expression as observed in microarray analysis. Overall, these observations revealed modulated expression of certain regulators involved in contending the abiotic stress, and one specific regulator of circadian function (Jmj30) in transgenic plants.

Our analysis also indicated that a group of defence related genes were down-regulated in transgenic plants in comparison to WT (Table 2). Quantitative RT-PCR analyses of these genes were in agreement with microarray results (Fig. 5). These genes comprised the pathogenesis related proteins (PR-1, PR-5), $\beta$-1,3-glucanase, chitinase, plant natriuretic peptide $A$, aspartyl protease family protein, crinkly related protein and ankyrin binding protein. A remarked decrease in transcript level of three cysteine rich receptor like protein kinases $(\mathrm{CRK})$ and two receptors like protein were observed among 28 down-regulated genes. PR proteins are, in 
A
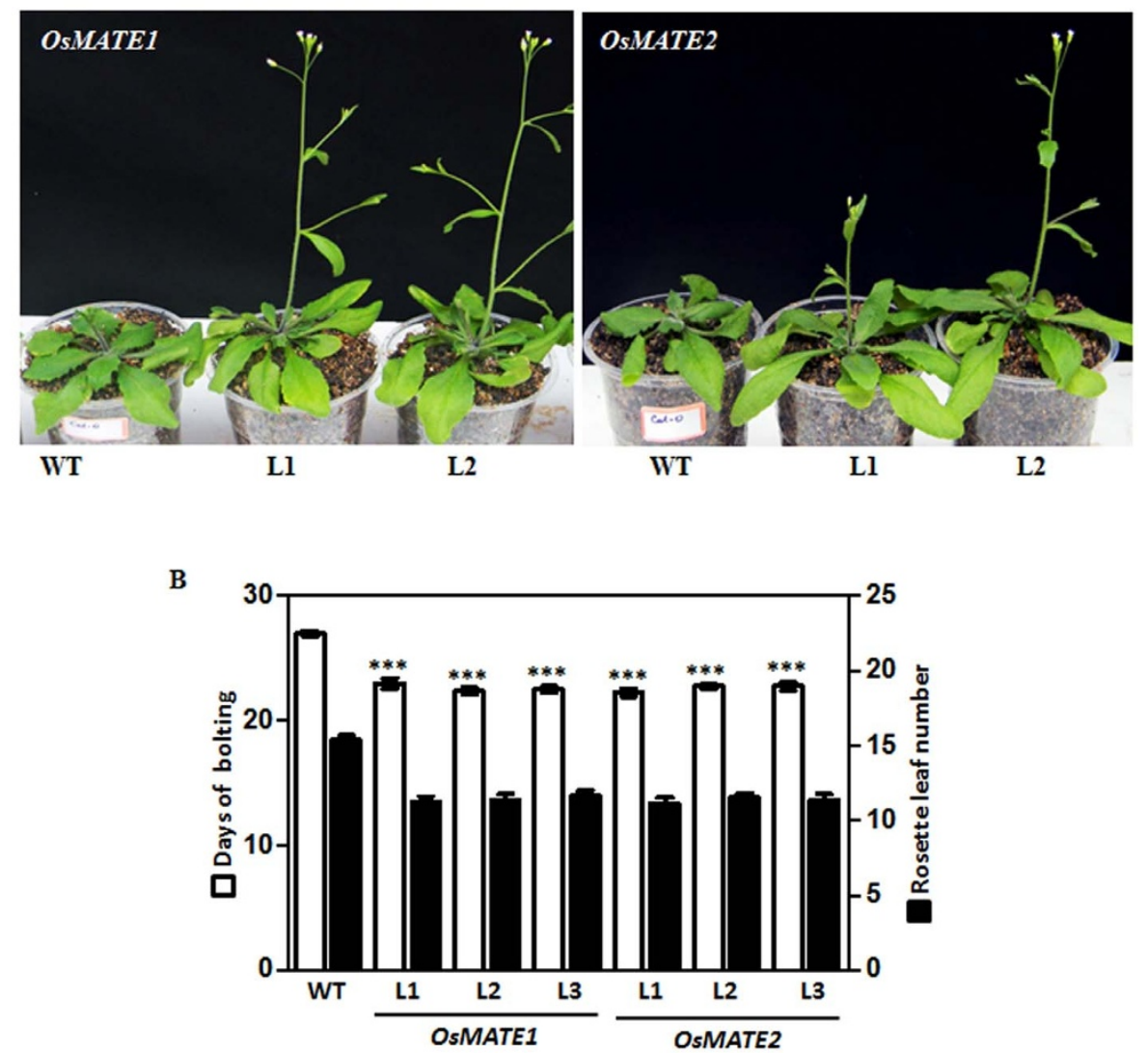

Figure $2 \mid$ Early flowering in transgenics lines expressing OsMATEs. (A) Expression of either of OsMATE1 or OsMATE2 in Arabidopsis induces early flowering in Arabidopsis. Photograph of two lines of OsMATE1 and OsMATE2 (nearly 3 week old) along with WT are displayed. (B) Number of rosette leaf and days at bolting of WT and 3 transgenic lines of the OsMATE1 and OsMATE2 grown under LD conditions (16 h light-8 h dark cycle). Data are the mean and \pm SE $(\mathrm{n}=8-12)$. Significance was evaluated by One-way ANOVA (Newman-Keuls used as post hoc test). ***Significantly different from WT $(\mathrm{P}<0.0001)$.

general, recruited downstream in salicylic acid signalling pathway and extend the cascading of signals ${ }^{9}$. Expression of $\beta$-1,3-glucanase, a class of PR proteins, and chitinase are induced during fungal infection that integrate a sort of tolerance while invasion of fungus in the plants $^{12}$. We conceived that the significantly reduced expression of stress related genes may lead to compromised defence response in transgenic plants.

Arsenic response of transgenic lines. Our earlier study suggested that OsMATE1 and OsMATE2 expression is enhanced during As exposure in rice. To examine the response of As on growth of OsMATEs expressing transgenic lines, seeds of transgenic lines, WT and pBI121 (empty vector transformed control) were grown for ten days on half strength MS containing agar plates with and without addition of As. All the genotypes grew similarly in absence of $\mathrm{As}(\mathrm{V})$. However, growth and root length of transgenic lines were significantly decreased in comparison to WT and pBI121 plants under As(V) rich environment (Supplementary Fig.S5). As participation of MATE proteins in combating the aluminium (Al) stress is well documented, the effects of $\mathrm{Al}$ on growth of transgenic lines were also evaluated. However, root length of all the genotypes was same at presence of $\mathrm{Al}$ in media and no change has been observed compared to WT and pBI121 plants (Supplementary Fig. S6). The root length data suggests that $\mathrm{As}(\mathrm{V})$ specifically sensitizes the growth of transgenic lines.

Enhanced disease susceptibility in transgenic lines. Expression analyses suggested that a group of defence related genes were down-regulated in transgenic plants compared to WT (Table 2). We envisioned that significantly reduced expression of stress related genes may lead to compromised defence response in transgenic plants. To further study the expression of MATEs under biotic stresses, we have investigated the expression pattern of OsMATE1 and OsMATE2 in Nipponbare and IAC165 cultivar of rice during infection of M. grisea isolate (FR13) and S. hermonthica using publically available microarray data ${ }^{36,37}$. Expression dataset exhibited that OsMATE1 was enhanced at post infection of M. grisea whereas its expression was repressed or unaffected during $S$. hermonthica interaction. By contrast, OsMATE2 induced specifically by the exposure of S. hermonthica (Fig. 6a). The expression analysis revealed that OsMATE1 and OsMATE2 were differentially expressed during $M$. grisea and $S$. hermonthica infections in rice and suggest that these genes might have a role in biotic stresses.

To ascertain the role of these MATEs in biotic stress, basal resistance of the transgenic and WT plants challenged with Pseudomonas syringeae (PstDC3000), a natural host of Arabidopsis causing spek disease was measured. After $3 \mathrm{dpi}$, an explicit enhanced susceptibility was observed in transgenic lines in comparison to WT in the form of the chlorotic lesion with a substantially higher bacterial titre (Fig. 6b, c). Our observations suggest that OsMATE1 and OsMATE2 may be negatively regulating disease tolerance apart from governing the growth and development. Furthermore, we have carried out infection assay after sprayed inoculation of bacterial culture on whole plants and quantified the bacterial titre at $7 \mathrm{dpi}$, which reconfirmed the susceptibility and significantly higher titre in all the transgenic lines expressing OsMATE1 and OsMATE2 (Supplementary Fig. S7). 
A

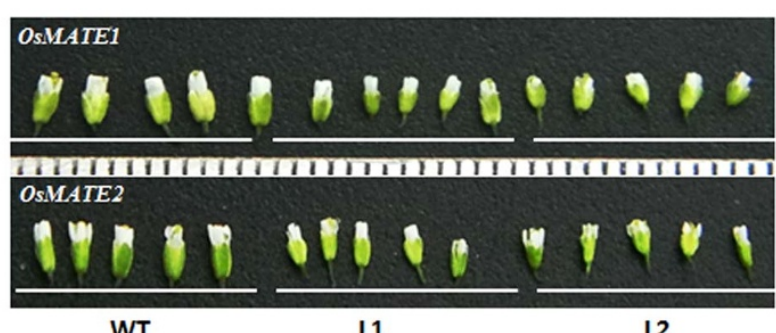

B

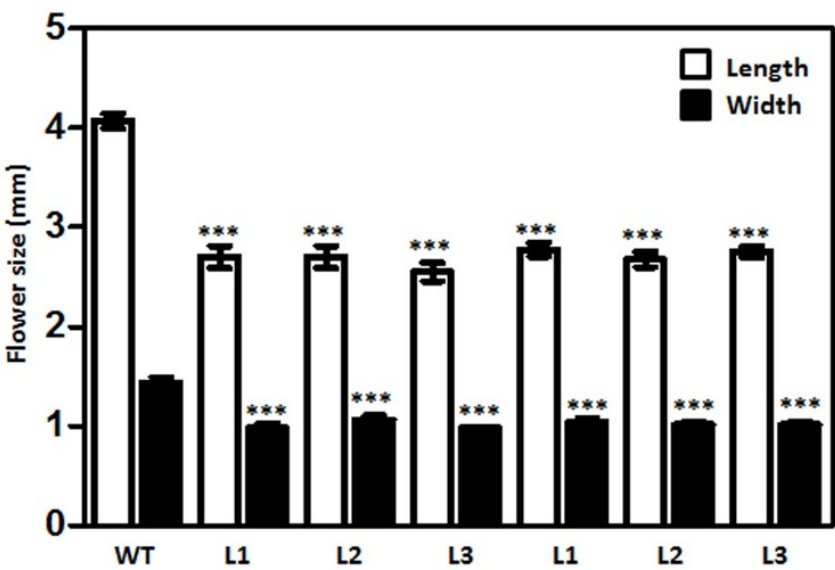

Figure 3 Variation of flower size in transgenic lines. (A) Flower size, both length and width, was reduced in transgenic plants. Photograph of flowers were taken after 5 week growth under standard conditions. (B) Bars are mean of length and width of 3 independent lines of each gene and variation represent as $\pm \mathrm{SE}(\mathrm{n}=8-12)$. Significance was evaluated by Oneway ANOVA (Newman-Keuls used as post hoc test). ***Significantly different from WT $(\mathrm{P}<0.0001)$.

Promoter of OsMATEs is active in seedling and flowering tissue. Expression of OsMATE1 and OsMATE2 in Arabidopsis led to several alterations related to plant growth and development. To establish function of these genes in detail, we studied tissue-specific expression of uidA gene under control of OsMATE1 and OsMATE2 promoters in Arabidopsis. In this regard, in silico analysis of promoters (ProMATE1, 1540 bp; ProMATE2, 1434 bp) has been carried out, which indicated the presence of various conserved consensus sequences of cis-regulatory elements. ProMATE1 has the 41 unique motifs which were absent in ProMATE2, Similarly, ProMATE2 contains 30 unique motifs (Fig. 7a). However, 36 common motifs were found in promoter region of both the genes (Fig. 7a). The detailed descriptions of these motifs are provided in Table S3, S4 and S5. Examination of GUS activity in all the tissues representing various developmental stages of Arabidopsis revealed a prominent GUS staining in newly formed leaves, shoot apex and in the root of ten days old seedlings for both OsMATE1 and OsMATE2 promoters (Fig. 7b). More importantly, no GUS activity was observed in other studied tissues (rosette and cauline leaves, stem) except in the reproductive tissues. After the fertilization, a strong GUS activity was seen in immature flower buds, early stage of embryo and seed development (Fig. 7b). Taken together, histological GUS staining of OsMATE1 and OsMATE2 promoters in Arabidopsis showed a cognate expression pattern, and suggests that reporter activity may be driven by the common motifs present in these promoters. Furthermore, a strong promoter activity at the shoot apex and during reproductive phase suggests the likely role of OsMATE1 and OsMATE2 in plant growth and development.

Sub-cellular localization of OsMATE1 and OsMATE2. In order to investigate the sub-cellular localization of OsMATE1 and OsMATE2, complete coding region of OsMATE1 and OsMATE2 were cloned in transient expression vector $326 \mathrm{sGFP}^{38}$ and transformed in protoplast isolated from mesophyll cells of Arabidopsis leaves. The examination of GFP fluorescence, after overnight incubation in dark, under confocal microscope explicitly suggests that OsMATE1 and OsMATE2 underlie on the plasma membrane, whereas a diffused green fluorescence was seen throughout the cell transformed with empty vector (Fig. 8). Altogether, these observations indicated that OsMATE1 and OsMATE2 reside on the plasma membrane.

Altered flavonoid content in transgenic lines. The relatedness of OsMATE1 and OsMATE2 with some of characterized flavonoid transporters in phylogenetic tree (Supplementary Fig. S1) suggest the possible role of these proteins in flavonoid transport. The two fold higher expression of GST (Glutathione-S transferase) in OSMATE1 expressing lines as observed in expression analyses also strengthen this hypothesis. The physical conjugation of glutathione to flavonoid catalysed by GST is a crucial metabolic step for flavonoids trafficking ${ }^{39-41}$. Therefore, content of major flavonols (kaempferol, quericetin and rutin) have been quantified in leaves of WT and transgenic lines. Flavonol profiling revealed the perturbation of kaempferol content in transgenic lines in comparison to WT plants. As speculated, kaempferol content was enhanced in transgenic leaves, which was undetectable in WT leaves (Table S6), and suggests that a proper flavonol channelling might be affected in transgenic plants.

\section{Discussion}

By using reverse genetics approach and a combination of molecular and biochemical tools, functional characterization of two members of the MATE family from rice, OsMATE1 and OsMATE2 has been carried out in this study. The scarcity of knowledge about the function of MATE proteins in rice, except the known citrate efflux activity of the FRDs ${ }^{29,30}$, encouraged us to ascribe the detailed role of OsMATEs out of total 53 proteins in rice genome. The constitutive expression of two As-responsive MATE encoding genes, OsMATE1 and OsMATE2, led to the diverse changes in growth and morphology of Arabidopsis. The major alterations were seen in leaf morphology, pattern of rosette leaves arrangement and flower development. We further demonstrate that transgenic lines were sensitive to $\mathrm{As}(\mathrm{V})$ exposure as well as exhibit a compromised basal resistance. Repressed immunity of transgenic lines was correlated with lowered expression of genes encoding pathogenesis related proteins.

Astonishingly, transgenic plants harbouring OsMATE1 and OsMATE2 independently displayed distinct common alterations that encompass leaf size, rosette leaf arrangement, flower, silique and seed morphology (Fig. 1-4). Nonetheless, these phenotypic variations were lacking in salk lines possibly due to presence of large members of this gene family with overlapping functions in Arabidopsis. For studying the mechanisms and responsible genes, we studied the genome wide expression analyses of transgenic and WT leaves. The analysis suggested that enhanced expression of a set of genes in transgenic plants may play important role in such phenotypic changes (Table 1 and 2). Two loci encoding the protein that contains NAC domain representing NAC transcription factor family were unregulated. NAC proteins constitute one of the largest families of transcription factors in plants and NAC domain containing transcription factors is considered as key regulator of stress perception and co-ordinating developmental programmes in plants ${ }^{42}$. Recently phytochrome-b interacting proteins, vascular plant one zinc finger 1 (VOZ1) and VOZ2, were identified to regulate the flowering in Arabidopsis. VOZ1 and VOZ2, a transcriptional activator, belong to the NAC family and voz1 voz2 double mutant showed a late flowering phenotype $\mathrm{e}^{43}$. In this study, we also reported an early flowering in transgenic lines and changes in silique number, flower and seed size corresponding to WT plants (Fig. 2-4). These changes might be due to increased transcript level of NAC transcription factors. Early 
A

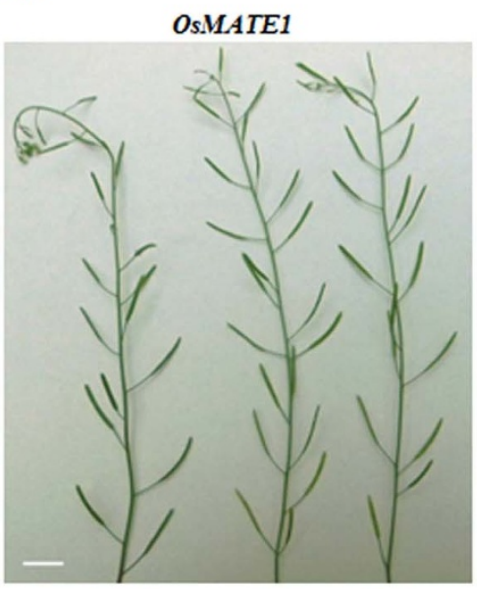

WT

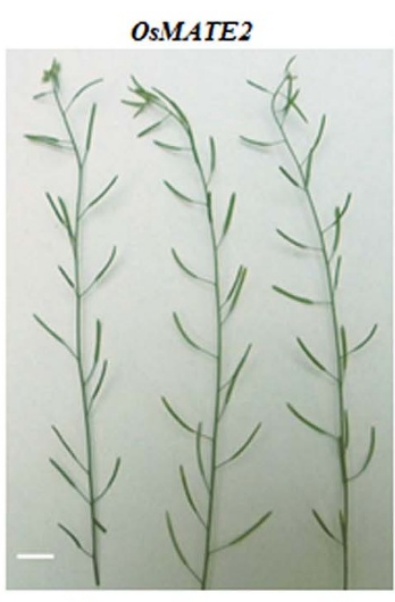

WT Ll

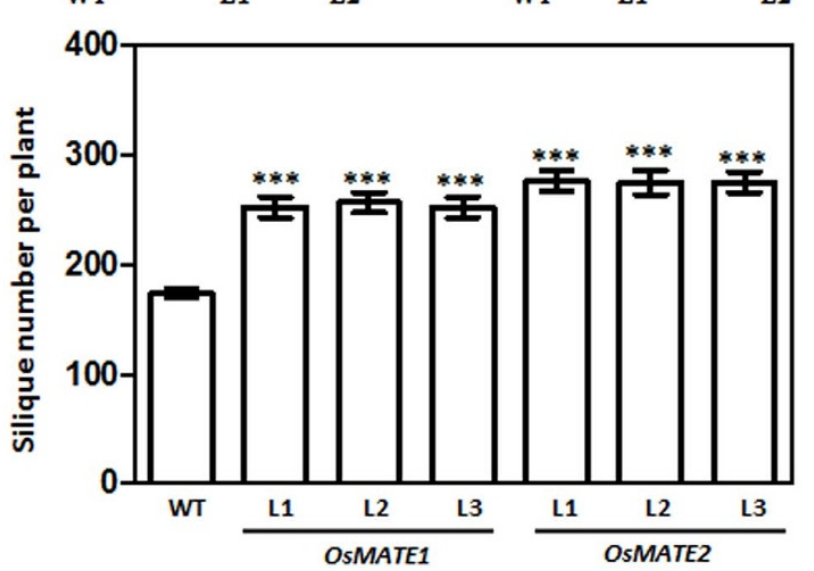

B

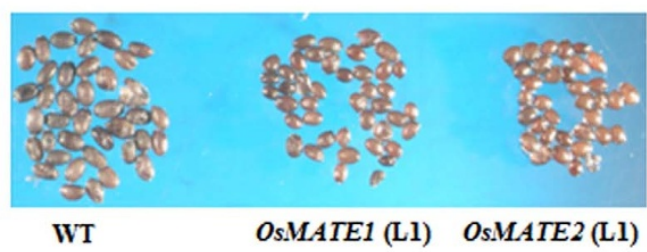

C
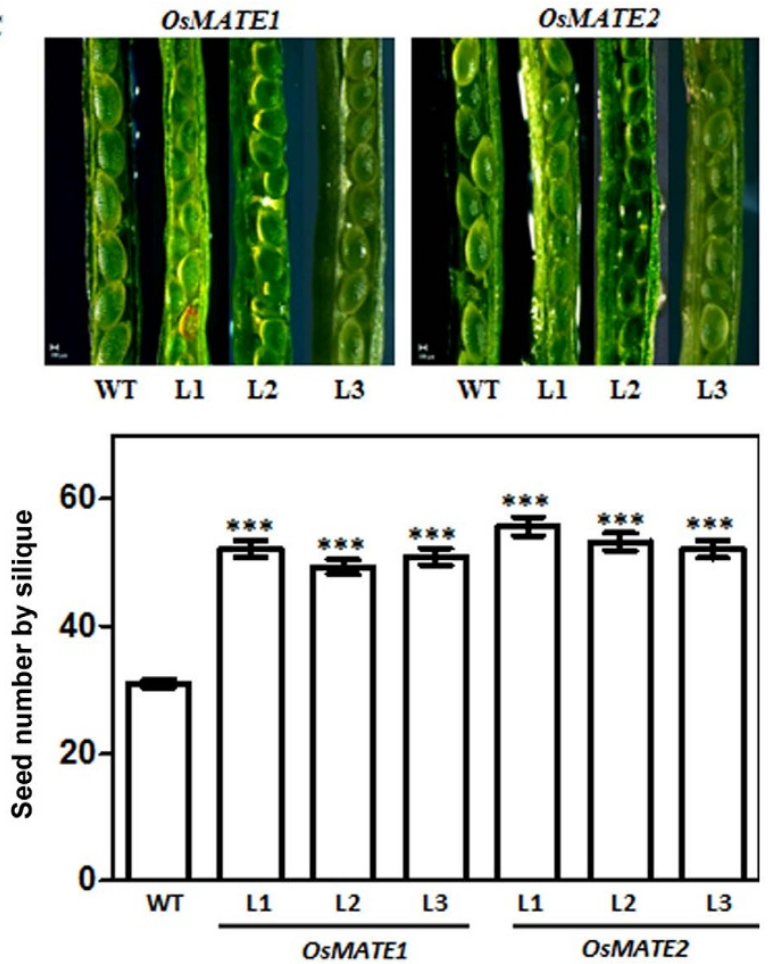

Figure $4 \mid$ Alteration in silique and seed number in transgenic plants. (A) All the transgenic lines more silique per plant than WT. Photograph of 6 week grown plants ( 2 lines of each transgenic) was taken. Data of 3 transgenic lines presented as mean and \pm SE $(n=8-12)$. (B) Seed size of transgenic and WT plants (representative of 1 line of each transgenic). (C) Seeds number per silique was high in transgenic lines. Values were collected from 10 silique per plant and 8-12 plant for each lines and WT. Data are presented as mean, \pm SE $(n=8-12)$. Significance was determined by One-way ANOVA (Newman-Keuls used as post hoc test). ***Significantly different from WT $(\mathrm{P}<0.0001)$.

flowering of transgenic plants can be due to enhanced expression of JumonjiC $(\mathrm{JmjC})$ containing protein. Basically, Jmj30 is a type of histone demethylase, which epigenetically regulated the circadian clock of Arabidopsis by affecting histone remodelling ${ }^{35}$. In
Arabidopsis, circadian clock regulates various processes such as gene expression, photoperiodic flowering, and leaf movement ${ }^{44}$. Thus, higher expression of $J m j 30$ might have a role in association with other factors as a determinant of early flowering in transgenic plants.

Table 1 | List of significantly up-regulated genes in OsMATEl expressing Arabidopsis lines

Locus ID

At5g59310

At5g39610

At3g20810

Atlg07590

At2g19970

At5g 17860

At2g33380

Atlg30260

AT2G29490

Atlg77450

At5g42900

At3g28290

At2g36120

At4g 15490

Atlg77760

At5g61820

Atlg72260

At5g23240
Annotation

LIPID TRANSFER PROTEIN 4

NAC domain containing protein 6

JUMONJI (jmiC) domain-containing protein

PENTATRICOPEPTIDE (ppr) repeat-containing protein

Pathogenesis related protein, putative

CAX7 (calcium exchanger 7)

RD20 (response to dessication20)

Unknown protein

Glutathione S-transferase TAU 1

NAC domain containing protein 32

Unknown protein

UPF0496 protein

Glycine-rich_protein

UDP-glycosyltransferase

Nitrate Reductase 1

Unknown protein

Toxin receptor binding (thionin2.1)

DNA heat shock $\mathrm{N}$-terminal domain-containing protein
Fold change

6.87

3.02

2.79

2.75

2.52

2.24

2.19

2.18

2.03

2.02

2.02

2

1.99

1.97

1.95

1.95

1.83

1.68 
Table 2 | List of significantly down-regulated genes in OsMATEl expressing Arabidopsis line

Locus ID

Annotation

Fold change

At2g14610

At3g57260

Atlg75040

At3g25010

At4g23150

At2g43570

At3g22600

Atlg 14870

At3g 18250

At2g18660

At5g10760

Atlg76960

At3g28540

At5g42830

At5g47850

At2g37430

At2g32680

Atlg74710

Atl g30900

At5g54610

A $+3 \mathrm{~g} 48650$

At4g23 140

Atlg35230

At3g 45860

At5g24530

Atlg35210

At3g27690

At2g32030

Pathogenesis related protein 1 (PR 1)

BETA-1,3-GLUCANASE 2 (BGL2)

Pathogenesis-related protein 5 (PR5)

$-57.81$

Receptor Like Protein 41 (RLK41)

$-7.76$

$-7.5$

Cysteine-rich RLK (CRK7)

Chitinase

Lipid transfer protein (LTP)

Plant $\mathrm{Cd}$ resistance protein

Membrane lipoprotein

Plant natriuretic peptide A (PNP-A)

Aspartyl protease family protein

Unknown protein

P-loop containing NTP hydrolases superfamily protein

$-6.57$

$-6.36$

$-5.9$

$-5.41$

$-4.87$

$-4.62$

$-4.34$

$-4.21$

$-3.6$

HXXXD-type acyl-transferase family protein

$-3.43$

CRINKLY4 RELATED 4(CCR4)

Zinc finger family protein (ZAT1 1)

$-3.33$

Receptor Like Protein 23 (RLP23)

$-3$

Isochorismate synthase 1 (ICS1)

VACUOLAR SORTING RECEPTOR 6 (VSR6)

ANK (ankyrin) protein binding

Unknown protein

CYSTEINE-RICH RLK 6 (CRK6)

ARABINOGALACTAN-PROTEIN 5(AGP5)

Cysteine-rich receptor-like protein kinase 4

Flavanone 3-hydroxylase-like protein

Unknown protein

Photosystem II light harvesting complex gene (LHCB2.3)

GCN5-related N-acetyltransferase (GNAT) family protein

$-2.99$

$-2.93$

$-2.86$

$-2.77$

$-2.62$

$-2.46$

$-2.42$

$-2.35$

$-2.02$

$-1.99$

$-1.97$

$-1.9$

$-1.81$

We further checked the co-expressed genes of Jmj30 by searching at ATTED-II to get a more clue at molecular level. Our analysis suggests that COL9 (At3g07650) and ELF4 (At2g40080) show a high correlation co-efficient along with Jmj30. COL9 belongs to CONSTANS gene family and is a negative regulator of photoperiodism and flowering via suppressing the expression of Flowering locus T (FT) and Suppressor of Overexpression of CO1 (SOC1) ${ }^{45}$. A subtle increase in expression of COL9 in transgenic lines supports the assumption (Supplementary Fig. S8). In addition, induced expression of the pentatricopeptide repeat containing (PPR) and other redox related genes suggest an imbalance of proper redox state of the cells.
There are few recent report emerges that correlate a synchrony between oxidative burst (ROS signalling) and circadian clock of Arabidopsis ${ }^{46}$.

Our expression analyses revealed that decreased expression of a group of defence related genes in OsMATE1 expressing Arabidopsis lines compared to WT (Table 2). These genes can be grouped in two broad categories, some of genes contribute to restrict the invasion of pathogen as their expression was highly induced, mainly comprising the PR proteins, $\beta-1,3-$ Glucanase and Receptor like proteins (Table 2). Proteins encoded by these genes play critical role in pathogen recognition and signal perception followed by activation of

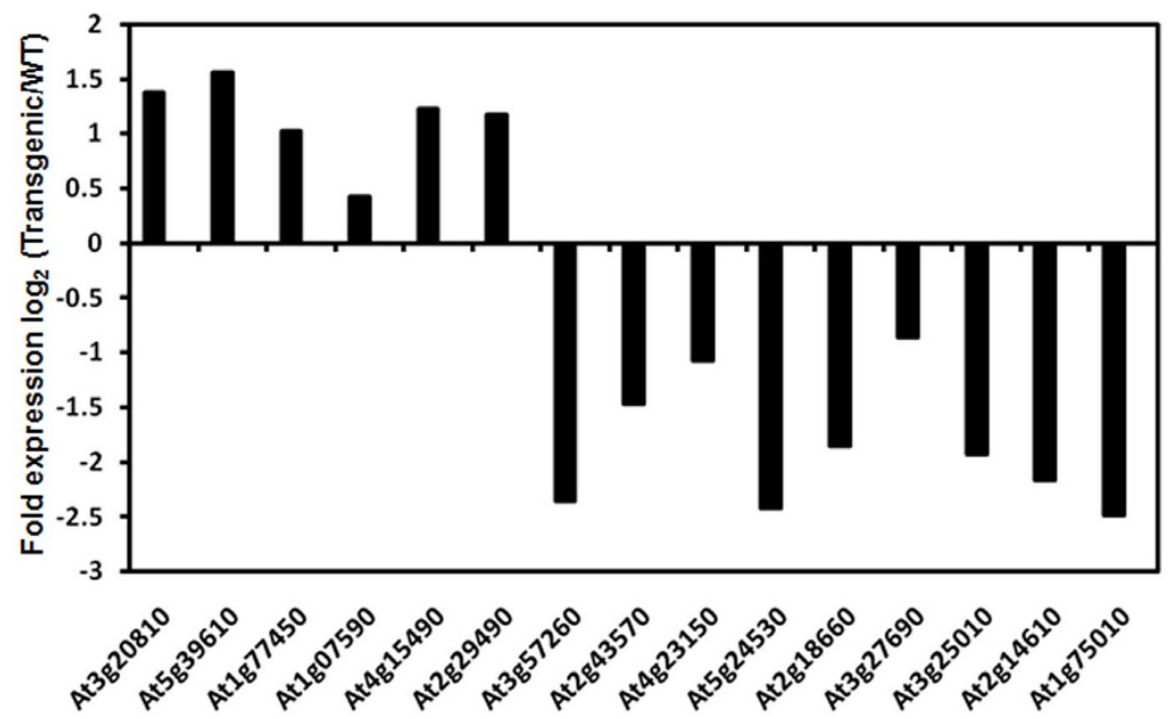

Figure $5 \mid$ Modulation of gene expression in transgenic line expressing OsMATE1. Relative gene expression between WT and transgenic plant was calculated using qRT-PCR ( tubulin was taken as endogenous control). Data provided represent fold expression $\log _{2}$ (transgenic/WT). Specific-primer set of individual genes was designed from intron spanning region. 


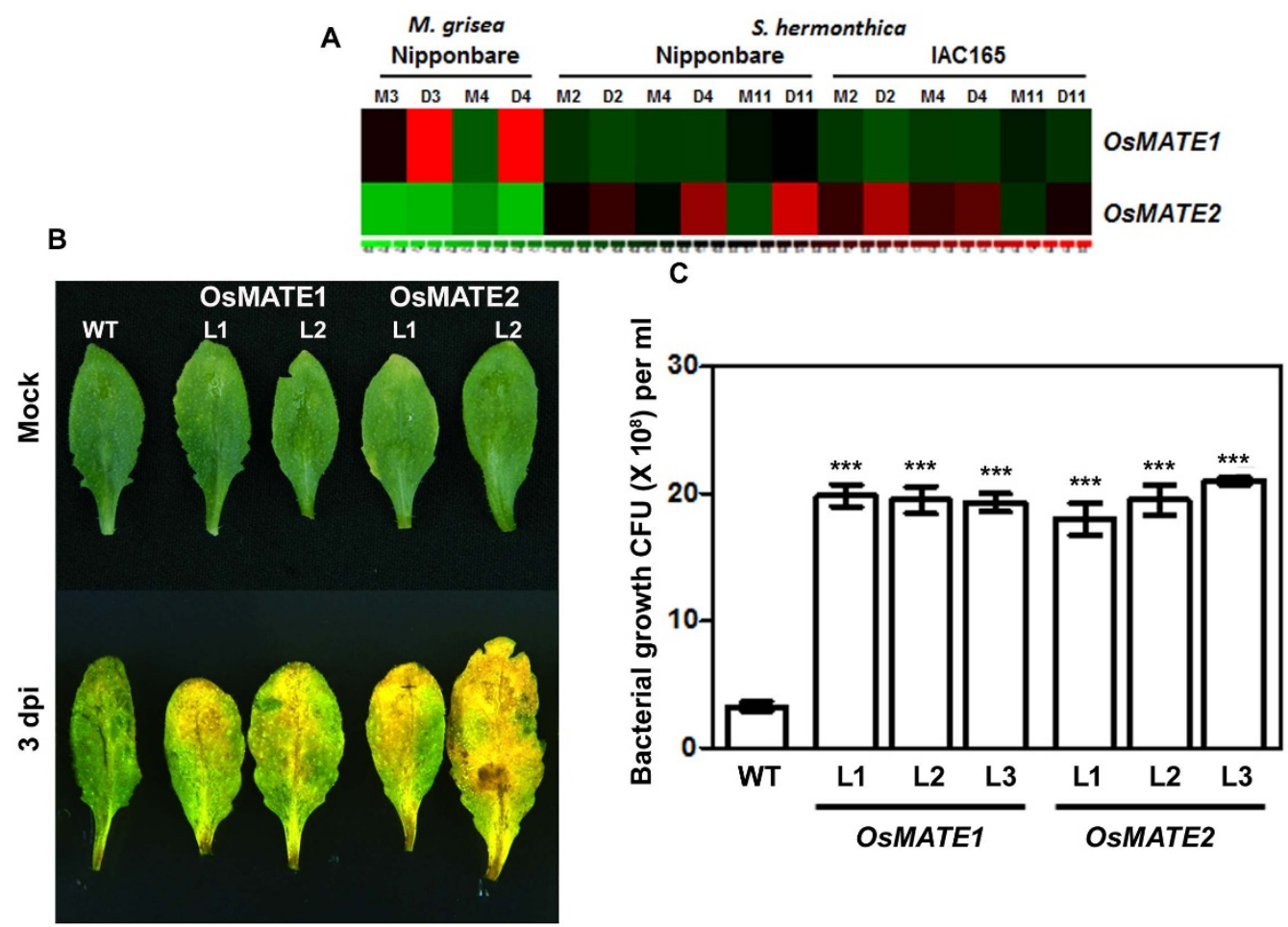

Figure 6 Bacterial infection assay. (A) Expression of OsMATE1 and OsMATE2 during infection of M. grisea on Nipponbare and S. hermonthica on Nipponbare and IAC165 cultivars of rice (Oryza sativa L.) at various indicated time, M2-M11 (mock) and D2-D11 (infection), days post inoculation of Affymetrix Rice Genome Array as cluster display. The colour scale (representing log signal values) is shown at the bottom. (B) Symptom of disease in leaves of WT and two transgenic lines at $3 \mathrm{dpi}$ after PstDC3000 spot inoculation. (C) Bacterial titre in the leaves was quantified at 3 dpi as shown as mean and standard errors calculated from three biological replicate (each leaf from different plants). The experiment was carried out more than three times. Significance was determined by One-way ANOVA (Newman-Keuls used as post hoc test). ${ }^{* * *}$ indicates significant difference (P $\left.<0.0001\right)$ as compared to WT.

defence responses. Similarly, cysteine-rich receptor like kinases and leucine rich repeat (LRR) protein kinases were significantly downregulated (Fig. 5). A NB-LRR is the major class of plant receptor, which intercepts effectors signal from much of pathogens and control the transcriptional mobilization of defence pathways in Arabidopsis ${ }^{47}$. Reduction in the expression of various genes responsible for signal perception to many downstream processes reflect the reduced basal disease resistance in transgenic plants and that was manifested in PstDC3000 infection assay as well (Fig. 6). An enhanced susceptibility was observed in transgenic lines expressing OsMATE1 and OsMATE2 compared to WT plants either on the detached leaf (Fig. 6b, c) or after seven dpi in planta sprays of bacterial inoculums (Supplementary Fig. S7). Similar to our observations, another study revealed that an activation tagging mutant of ads1-D (MATE protein) is highly susceptible to PstDC3000 and knockdown of ADS1 results a higher basal resistance in Arabidopsis $^{12}$. A systematic proteomic analysis of root exudates of Arabidopsis revealed that chitinase, $\beta-1,3-G$ lucanase and other defence proteins are secreted in higher amount during flowering and shown to link between flowering and defence processes ${ }^{48}$. The involvement of MATE proteins in disease resistance is unexceptionally accepted, and formerly identified during genetic screen of Arabidopsis mutants ${ }^{9,12}$ however underpinning molecular mechanism is explored very recently ${ }^{10,11}$. It has been revealed that EDS5 regulate salicylic acid biosynthesis by exporting from chloroplast. Flavonoids are often regarded as prominent signalling compound and interplay during biotic stresses however underlying actual molecular events of flavonoids signalling is not very much known. It has been reported that through managing accumulation of some flavonols plants can better adapt under plenty of pathogens and insect attacks ${ }^{49,50}$. In our study, flavonoid profiling of transgenic and WT leaves suggested altered flavonol content in transgenic plants in comparison to WT plants. We propose that perturbation of kaempferol, through OsMATEs expression in Arabidopsis, could be the central and foremost event that persuades morphological alterations. Although, specific substrates transported by these transporters have not resolved yet, but the results presented here indicated that it may be a flavonol, and which need to explore in future by molecular transport experimentation.

A prominent (AsV) sensitivity was observed in transgenic lines expressing MATEs as compared to WT plants (Supplementary Fig. S5). The sensitivity of transgenic plants could be explained on the basis of inevitable contribution of flavonoids in maintaining redox by retaining ROS scavenging activity. Disturbance of this process via perturbation of intracellular flavonoids either at different steps of their biosynthesis or transport could lead to the manifestation of $\mathrm{As}(\mathrm{V})$ sensitive phenotype. Plasma-membrane localized MATE transporters are devoted for citrate efflux while others are recognised for flavonoid and other secondary metabolites sequestration in vacuoles $^{25,30,51}$. The previously identified role of MATE transporters engage in flavonoids sequesteration are found to mostly restricted on vacuolar membrane rather than plasma-membrane localization of OsMATE1 and OsMATE2, which is elucidated in this study (Fig. 8). Thus, more and more information generated about functional significance of members of this gene family in plants, would elaborate our insight about transport of flavonoids and other compounds ranging from intracellular to long distance transport and their impact on various physiological processes in future.

In conclusion, our study suggests that OsMATE1 and OsMATE2 are functionally redundant to each other based on observations obtained in transgenic lines as both MATEs direct the common phenotypic changes through expression in Arabidopsis as well as 


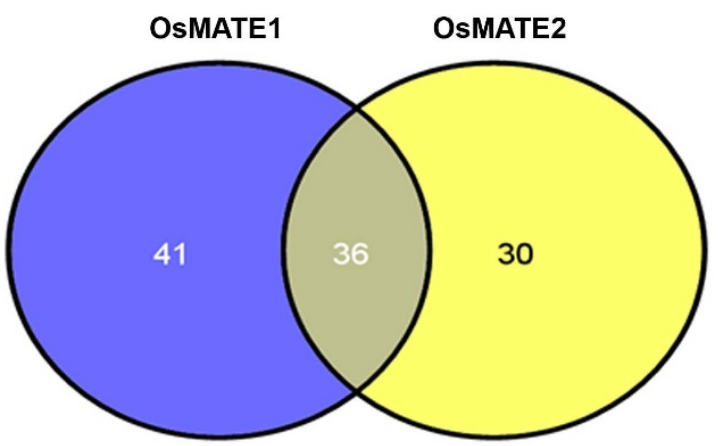

B
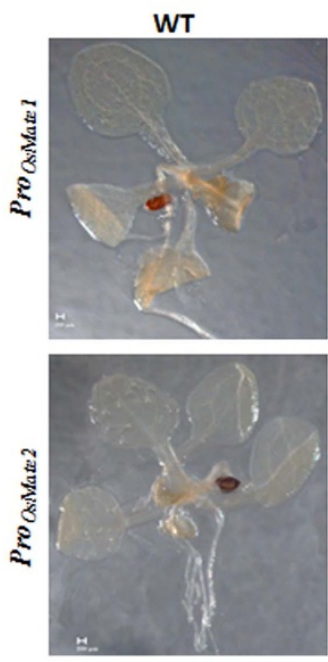

L1
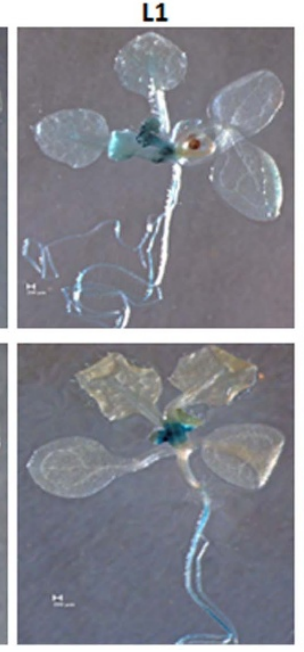

L2
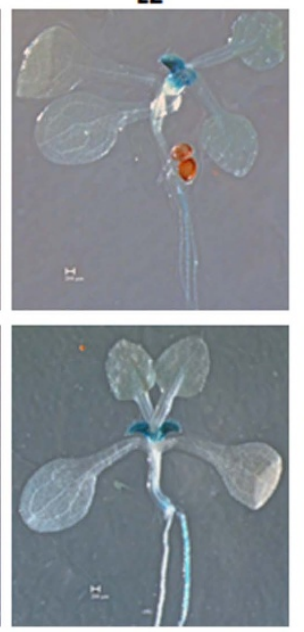

Flowering buds (L1)
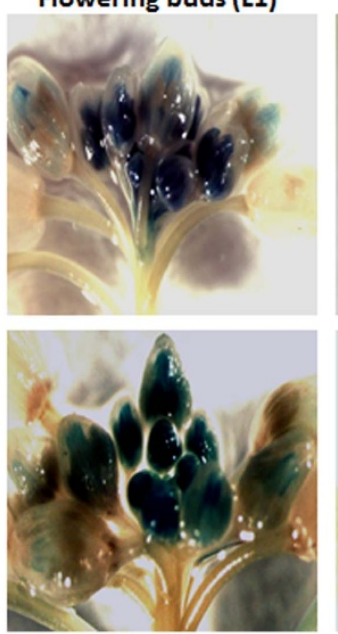

Silique
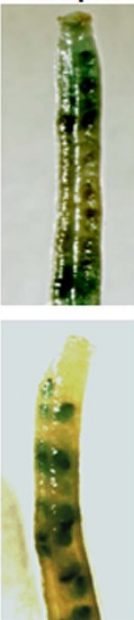

Figure $7 \mid$ Promoter analyses of OsMATE1 and OsMATE2. (A) cis-regulatory elements of ProOsMATE1 and ProOsMATE2 was analysed through PLACE and venn diagram shows the number of common and unique motifs present in promoters. (B) Promoter activity in WT and Arabidopsis lines expressing ProOsMATE1:uidA and ProOsMATE2:uidA. Representatives of GUS staining performed in seedling (10 day old), flowers and silique of mature plant. White scale bars present in each panel in (B) are equivalent to $100 \mu \mathrm{m}$ length.

their similar promoter activities in Arabidopsis. There is a necessity of thorough study in the future to obtain more insight about nature of substrates transported by these proteins to establish a direct link between growth-development and disease resistance in plants.

\section{Methods}

Plant material and growth condition. Arabidopsis thaliana (L.) accession Col- 0 and salk mutants (SALK_045655C and SALK_124549C) were used in the study. Arabidopsis plants were grown in growth chamber (Canviron, USA) under controlled conditions $16 \mathrm{~h}$ light $-8 \mathrm{~h}$ dark photoperiod cycle, $22^{\circ} \mathrm{C}$ temperature, $150-180 \mu \mathrm{mE}$ $\mathrm{m}^{-2} \mathrm{~s}^{-1}$ light intensity and $60 \%$ relative humidity. Seeds were sown in soilrite and stratified for $48 \mathrm{~h}$ in the dark at $4^{\circ} \mathrm{C}$ before shifting in the light. Plants were watered with nutrient media $\left(5 \mathrm{mM} \mathrm{KNO}_{3}, 2 \mathrm{mM} \mathrm{MgSO}_{4}, 2 \mathrm{mM} \mathrm{Ca}\left(\mathrm{NO}_{3}\right)_{2}, 2 \mathrm{mM} \mathrm{K}\right.$ $\mathrm{H}_{2} \mathrm{PO}_{4}, 50 \mu \mathrm{M} \mathrm{Fe}(\mathrm{II})$. EDTA, $70 \mu \mathrm{M} \mathrm{H}_{3} \mathrm{BO}_{3}, 14 \mu \mathrm{M} \mathrm{MnCl}_{2}, 1 \mu \mathrm{M} \mathrm{ZnSO}_{4}, 0.5 \mu \mathrm{M}$ $\mathrm{CuSO}_{4}, 0.2 \mu \mathrm{M} \mathrm{Na}_{2} \mathrm{MoO}_{4}, 10 \mu \mathrm{M} \mathrm{NaCl}, 0.01 \mu \mathrm{M} \mathrm{CoCl}_{2}$ ) and reverse osmosis (RO) water at alternate interval in every week until plants complete their lifecycle.

Database search and Bioinformatics analysis. MATE sequences were searched from the database on the BLAST server (http://www.ncbi.nlm.nih.gov/blast/Blast.cgi and http://rice.plantbiology.msu.edu) by using keyword, domain name and BLAST against characterized Arabidopsis MATEs. We retrieved full length MATE sequences of rice, Arabidopsis, and fully characterized MATE proteins from other organisms. Multiple amino acid sequences were aligned with the help of ClustalW alignment tool (http://www.ebi.ac.uk/Tools/clustalw2/index.html). The phylogenetic tree using fulllength MATEs was constructed by the neighbour-joining method using Phylip ${ }^{52}$ and displayed using PhyloDraw V0.82 $2^{53}$. Expression study of OsMATE1 and OsMATE2 during biotic stress in rice has been carried out using publicly available microarray CEL files in the GEO database. GEO database for virulent infection by Magnaporthe grisea (GSE7256) and expression data for interaction with the plant parasite Striga hermonthica (GSE10373) were used in expression analysis. The Affymetrix CEL files of each experiment were imported and analysed using d-Chip under default setting ${ }^{54}$.

Cloning, construct preparation and transgenic plant development. Full-length cDNAs of OsMATE1 and OsMATE2 were amplified from the single stranded cDNA library prepared from rice root though PCR by using nested primers (Table S1) and cloned in $\mathrm{pT} / \mathrm{Z}$ subcloning vector (Fermentas, USA). Another set of primers was designed with restriction sites of $\mathrm{Xba} 1$ and Sacl enzymes in forward and reverse primers, respectively. The coding region of both MATEs was cloned in plant expression vector pBI121 between $X b a 1$ and Sac1 restriction sites. These constructs were transformed in Agrobacterium tumefaciens strain GV3101 by the freeze thaw method. Arabidopsis transgenic plants were generated through floral dip method ${ }^{55}$. Transformants were screened on half MS agar plates containing kanamycin $(50 \mu \mathrm{g}$ per ml media). Based on the relative expression of transgene through semi quantitative RT-PCR, three lines for each of OsMATE1 and OsMATE2 among the 15 screened independent lines were selected for further analysis. All the studies were performed in T4 homozygous lines. For promoter analysis, upstream sequences from the translation initiation codon of OSMATE1 (1540 bp) and OSMATE2 (1434 bp) were retrieved and amplified using High Fidelity enzyme mix (Fermentas, USA) from genomic DNA and cloned in pCambia1303 plant binary vector between HindIII and $\mathrm{NcoI}$ restriction sites. Transgenic plants were generated and screened on hygromycin $(10 \mu \mathrm{g}$ per $\mathrm{ml})$. Similarly, for preparing the construct employed in sub-cellular localization, coding region of OSMATE1 and OsMATE2 were cloned in 326 sGFP

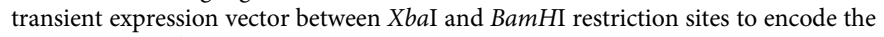
fusion proteins $s^{56}$. All the clones were sequenced to ensure the in frame fusion and error free preparation of constructs.

Microarray and gene expression analysis. Microarray analysis was performed using RNA isolated from rosette leaves of nearly three weeks old Col-0 and OsMATE1 expressing plants. Whole genome Arabidopsis ATH1 array (Affymetrix, USA) was utilized in experiment and each step was followed as per manufacturer's instruction. Total RNA was isolated from rosette leaves by using Spectrum Plant Total RNA Kit (Sigma, USA), followed by treatment with RNAase-free DNase (Fermentas, USA) to remove the genomic DNA. Briefly, $250 \mathrm{ng}$ total RNA of each sample was taken as starting material and strictly followed the Affymetrix protocol during RNA amplification, hybridization, washing-staining and scanning of the arrays. Normalization of cell files and relative fold change were measured using d-Chip ${ }^{54}$. Quantitative Real time PCR (qRT-PCR) was carried out to validate microarray data. Total RNA $(2 \mu \mathrm{g})$ was used in first strand cDNA library preparation (RevertAid H minus first strand cDNA synthesis Kit Fermentas, USA). One $\mu$ l template was taken 

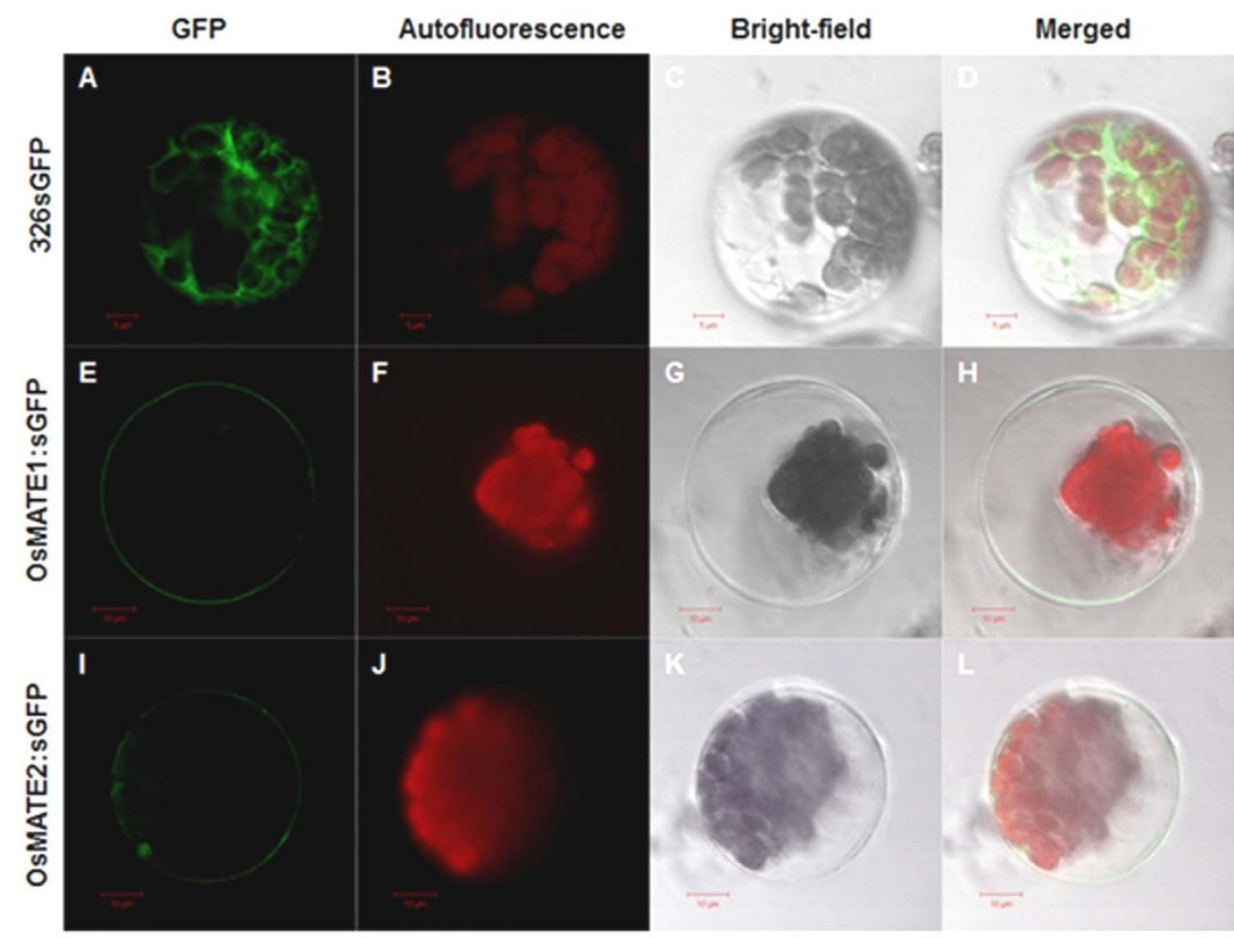

Figure 8 OsMATE1 and OsMATE2 reside on plasma-membrane. Arabidopsis mesophyll protoplast transformed with 326sGFP (A-D), OsMATE1:GFP (E-H) and OsMATE2:GFP (I-L). Confocal cross sections show GFP fluorescence (excitation $488 \mathrm{~nm}$ and emission light measured through 505-530 nm band pass filter), autofluorescence of cell (excitation $543 \mathrm{~nm}$, emission measured between $580 \mathrm{~nm}$ and $705 \mathrm{~nm}$ ), bright field images and their overlay in different panels. (D) (H) and (I) represent overlay of (A-C), (E-G) and (I-K), respectively. Scale bars are included in images.

in each reaction after 10 times dilution with nuclease free water. A reaction was setup with template, forward primer, reverse primer and Fast syber green mix in AB Fast 7500 thermal cycler (Applied Biosystems, UK). Unique primer set (MWG, India) was designed from the intron spanning region of each studied gene, wherever possible, and tubulin was taken as endogenous control to normalize the data (Supplementary Table S1). For semi-quantitative RT PCR analysis, first strand cDNA was taken as a template and a primer set specific for OsMATE1 and OsMATE2 (Table S1) and $2 \times$ master mix (Fermentas, USA) were used. A reaction set up, with an initial denaturation at $94^{\circ} \mathrm{C}$ for $2 \mathrm{~min}, 28$ cycles at $94^{\circ} \mathrm{C}$ for $15 \mathrm{~s}, 55^{\circ} \mathrm{C}$ for $15 \mathrm{~s}$, and $72^{\circ} \mathrm{C}$ for $30 \mathrm{~s}$, followed by a final $5 \mathrm{~min}$ extension at $72^{\circ} \mathrm{C}$, condition were executed in $20 \mu \mathrm{l}$ reaction volume. PCR products were separated in $1.2 \%$ agarose gel in $0.5 \times \mathrm{TBE}$ running buffer. For taking equal amount of cDNA tubulin was taken as an endogenous control.

Metal exposure and root growth assay. Seeds of all the genotypes were surface sterilized with sodium hypochlorite and plated on half strength MS agar plates with and without supplementation of $\mathrm{As}(\mathrm{V})$ and $\mathrm{Al}^{57}$. Plates were kept in dark for $48 \mathrm{~h}$ at $4^{\circ} \mathrm{C}$ (stratification) and photographed after the growth of ten days in standard growth conditions. Root lengths of each genotype and WT plants were manually measured and taken from at least three separate plates.

Analyses of transgenic lines. Homogygous transgenic lines expressing OsMATE1 and OsMATE2 were grown for complete life cycle and all observations regarding different phenotypic characters were collected at indicated growth stages and monitored at least 2-3 successive generations. For histological GUS activity, ProOsMATE1:uidA and ProOsMATE2:uidA transgenic lines were grown on half strength MS agar plates for 10 days or on soilrite. Seedlings and different tissues from mature plants were rinsed in ice cold PBS buffer 3-4 times and after three washing samples were incubated $16 \mathrm{~h}$ at $37^{\circ} \mathrm{C}$ in GUS staining buffer containing $100 \mathrm{mM}$ $\mathrm{NaPO}_{4}, 1.0 \mathrm{mM}$ ferrocyanide, $1.0 \mathrm{mM}$ ferricyanide, $0.05 \%$ Triton X-100, pH 7.2, and $2 \mathrm{mM}$ 5-bromo-4-chloro-3-indolyl-b-D-glucuronide (initially dissolved in $\mathrm{N}, \mathrm{N}$ Dimethyl formamide $)^{58}$. After successive washing from the gradient of ethanol $(70 \%-$ 90\%), images were captured under light microscope (Carl Zeis, USA).

Bacterial infection assay. Pseudomonas syringae pv. tomato strain (PstDC3000) was allowed to grow overnight in king's B media containing $15 \mu \mathrm{g}$ rifampicin per $\mathrm{ml}$ media at $28^{\circ} \mathrm{C}$ with $220 \mathrm{rpm}$ shaking. Cells were collected by $5000 \mathrm{rpm}$ centrifugation and washed with sterile $0.8 \%$ gelatin. Bacterial OD was adjusted to $5 \times$ $10^{8} \mathrm{cfu} / \mathrm{ml}$ in $0.8 \%$ gelatin for detach leaf infection assay. Leaves of three week old transgenic lines and Col- 0 were surface sterilized with $0.04 \% \mathrm{NaOCl}$ and washed with autoclaved water several times. Finally, two spot of $10 \mu \mathrm{l}$ bacterial inoculum was applied on each leaf and incubated at $28^{\circ} \mathrm{C}$ for $48 \mathrm{~h}$ to establish the infection and bacterial cfu was then determined. For in planta spray assay, bacterial inoculum was adjusted to $5 \times 10^{5} \mathrm{cfu} / \mathrm{ml}$ in $0.8 \%$ gelatin and sprayed on whole plants equally. Infected plants were kept in growth chamber under $16 \mathrm{~h}$ light- 8 h dark cycle at $22^{\circ} \mathrm{C}$ for 7 days and then bacterial titres were measured.

Sub-cellular localization. For sub-cellular localization, complete coding sequences of OSMATE1 and OsMATE2 were fused upstream, in frame with, to $\mathrm{SGFP}^{38}$ under control of CaMV $35 \mathrm{~S}$ promoter in $326 \mathrm{sGFP}$ vector ${ }^{56}$. These constructs were transiently expressed in protoplasts isolated from mesophyll cells of four weeks old Arabidopsis leaves and $15 \mu \mathrm{g}$ of purified DNA was used in polyethylene glycol mediated transformation ${ }^{59}$. After overnight incubation transient expression of GFP fusion protein was examined. Images were captured under a confocal microscope (LSM 510, Zeiss, USA).

Flavonoids estimation. For flavonoids quantification, individual flavonols in the rosette leaves were determined by methods of Misra et al. (2010). Briefly, plant material $\left(500 \mathrm{mg}\right.$ ) was grinded finely in liquid $\mathrm{N}_{2}$ and extracted in $5 \mathrm{ml}$ methanol: water $(80: 20)$ with constant agitation for $2 \mathrm{~h}$ and supernatant was collected after centrifugation. Pellet was re-extracted in $5 \mathrm{ml}$ methanol: water for overnight at room temperature with constant agitation and supernatant was filtered. Both the filtrates were pooled, concentrated in rotavapour and again dissolved in $1.0 \mathrm{ml}$ of $80 \%$ methanol. Separation for qualitative and quantitative analysis of flavonols was performed by HPLC-PDA with a Shimadzu (Kyoto, Japan) LC-10 system comprising an LC-10 AT dual pump system, an SPD-M20A PDA detector $(254 \mathrm{~nm})$ and rhodyne injection valve furnished with a $20 \mu \mathrm{l}$ sample loop.

Statistical analysis. Each experiment was carried out under a completely randomized design with at least three replications. Phenotypic observations were measured upto 2-3 generations for consistency. Data are expressed as mean \pm SEM and subjected to one-way ANOVA followed by post hoc Newman-Keuls for multiple comparison test of significance using GraphPad Prism 3.02.

1. Kuroda, T. \& Tsuchiya, T. Multidrug efflux transporters in the MATE family. Biochim Biophys Acta 1794, 763-768 (2009).

2. Morita, Y. et al. NorM, a putative multidrug efflux protein, of Vibrio parahaemolyticus and its homolog in Escherichia coli. Antimicrob Agents Chemother 42, 1778-1782 (1998).

3. He, X. et al. Structure of a cation-bound multidrug and toxic compound extrusion transporter. Nature 467, 991-994 (2010).

4. Lu, M. et al. Structures of a $\mathrm{Na}+$-coupled, substrate-bound MATE multidrug transporter. Proc Natl Acad Sci U S A 110, 2099-2104 (2013). 
5. Zhao, J. \& Dixon, R. A. MATE transporters facilitate vacuolar uptake of epicatechin 3'-O-glucoside for proanthocyanidin biosynthesis in Medicago truncatula and Arabidopsis. Plant Cell 21, 2323-2340 (2009).

6. Hvorup, R. N. et al. The multidrug/oligosaccharidyl-lipid/polysaccharide (MOP) exporter superfamily. Eur J Biochem 270, 799-813 (2003).

7. Diener, A. C., Gaxiola, R. A. \& Fink, G. R. Arabidopsis ALF5, a multidrug efflux transporter gene family member, confers resistance to toxins. Plant Cell 13, 1625-1638 (2001).

8. Li, L., He, Z., Pandey, G. K., Tsuchiya, T. \& Luan, S. Functional cloning and characterization of a plant efflux carrier for multidrug and heavy metal detoxification. J Biol Chem 277, 5360-5368 (2002).

9. Nawrath, C., Heck, S., Parinthawong, N. \& Metraux, J. P. EDS5, an essential component of salicylic acid-dependent signaling for disease resistance in Arabidopsis, is a member of the MATE transporter family. Plant Cell 14, 275-286 (2002)

10. Yamasaki, K. et al. Chloroplast envelope localization of EDS5, an essential factor for salicylic acid biosynthesis in Arabidopsis thaliana. Plant Signal Behav 8 (2013).

11. Serrano, M. et al. Export of salicylic acid from the chloroplast requires the multidrug and toxin extrusion-like transporter EDS5. Plant Physiol 162 1815-1821 (2013).

12. Sun, X. et al. ADS1 encodes a MATE-transporter that negatively regulates plant disease resistance. New Phytol 192, 471-482 (2011).

13. Magalhaes, J. V. et al. A gene in the multidrug and toxic compound extrusion (MATE) family confers aluminum tolerance in sorghum. Nat Genet 39, 1156-1161 (2007).

14. Furukawa, J. et al. An aluminum-activated citrate transporter in barley. Plant Cell Physiol 48, 1081-1091 (2007)

15. Liu, J., Magalhaes, J. V., Shaff, J. \& Kochian, L. V. Aluminum-activated citrate and malate transporters from the MATE and ALMT families function independently to confer Arabidopsis aluminum tolerance. Plant J 57, 389-399 (2009).

16. Ryan, P. R., Raman, H., Gupta, S., Horst, W. J. \& Delhaize, E. A second mechanism for aluminum resistance in wheat relies on the constitutive efflux of citrate from roots. Plant Physiol 149, 340-351 (2009).

17. Maron, L. G. et al. Two functionally distinct members of the MATE (multi-drug and toxic compound extrusion) family of transporters potentially underlie two major aluminum tolerance QTLs in maize. Plant J 61, 728-740 (2010).

18. Maron, L. G. et al. Aluminum tolerance in maize is associated with higher MATE1 gene copy number. Proc Natl Acad Sci U S A 110, 5241-5246 (2013).

19. Lepiniec, L. et al. Genetics and biochemistry of seed flavonoids. Annu Rev Plant Biol 57, 405-430 (2006).

20. Debeaujon, I., Peeters, A. J., Leon-Kloosterziel, K. M. \& Koornneef, M. The TRANSPARENT TESTA12 gene of Arabidopsis encodes a multidrug secondary transporter-like protein required for flavonoid sequestration in vacuoles of the seed coat endothelium. Plant Cell 13, 853-871 (2001).

21. Marinova, K., Kleinschmidt, K., Weissenbock, G. \& Klein, M. Flavonoid biosynthesis in barley primary leaves requires the presence of the vacuole and controls the activity of vacuolar flavonoid transport. Plant Physiol 144, 432-444 (2007)

22. Zhao, J. et al. MATE2 mediates vacuolar sequestration of flavonoid glycosides and glycoside malonates in Medicago truncatula. Plant Cell 23, 1536-1555 (2011).

23. Gomez, C. et al. Grapevine MATE-type proteins act as vacuolar $\mathrm{H}+$-dependent acylated anthocyanin transporters. Plant Physiol 150, 402-415 (2009).

24. Morita, M. et al. Vacuolar transport of nicotine is mediated by a multidrug and toxic compound extrusion (MATE) transporter in Nicotiana tabacum. Proc Natl Acad Sci U S A 106, 2447-2452 (2009).

25. Shoji, T. et al. Multidrug and toxic compound extrusion-type transporters implicated in vacuolar sequestration of nicotine in tobacco roots. Plant Physiol 149, 708-718 (2009).

26. Durrett, T. P., Gassmann, W. \& Rogers, E. E. The FRD3-mediated efflux of citrate into the root vasculature is necessary for efficient iron translocation. Plant Physiol 144, 197-205 (2007).

27. Fernandez, J. et al. Principles of carbon catabolite repression in the rice blast fungus: Tps1, Nmr1-3, and a MATE-family pump regulate glucose metabolism during infection. PLoS Genet 8, e1002673 (2013).

28. Burko, Y. et al. From organelle to organ: ZRIZI MATE-Type transporter is an organelle transporter that enhances organ initiation. Plant Cell Physiol 52, 518-527 (2011).

29. Yokosho, K., Yamaji, N., Ueno, D., Mitani, N. \& Ma, J. F. OsFRDL1 is a citrate transporter required for efficient translocation of iron in rice. Plant Physiol 149, 297-305 (2009).

30. Yokosho, K., Yamaji, N. \& Ma, J. F. An Al-inducible MATE gene is involved in external detoxification of $\mathrm{Al}$ in rice. Plant J 68, 1061-1069 (2011).

31. Chakrabarty, D. et al. Comparative transcriptome analysis of arsenate and arsenite stresses in rice seedlings. Chemosphere 74, 688-702 (2009).

32. Yuan, H. \& Liu, D. Functional disruption of the pentatricopeptide protein SLG1 affects mitochondrial RNA editing, plant development, and responses to abiotic stresses in Arabidopsis. Plant J 70, 432-444 (2012).

33. Laluk, K., Abuqamar, S. \& Mengiste, T. The Arabidopsis mitochondria-localized pentatricopeptide repeat protein PGN functions in defense against necrotrophic fungi and abiotic stress tolerance. Plant Physiol 156, 2053-2068 (2011).
34. Park, J. et al. Integration of auxin and salt signals by the NAC transcription factor NTM2 during seed germination in Arabidopsis. Plant Physiol 156, 537-549 (2011).

35. Lu, S. X. et al. The Jumonji C domain-containing protein JMJ30 regulates period length in the Arabidopsis circadian clock. Plant Physiol 155, 906-915 (2011).

36. Ribot, C. et al. Susceptibility of rice to the blast fungus, Magnaporthe grisea. J Plant Physiol 165, 114-124 (2008).

37. Swarbrick, P. J. et al. Global patterns of gene expression in rice cultivars undergoing a susceptible or resistant interaction with the parasitic plant Striga hermonthica. New Phytol 179, 515-529 (2008).

38. Niwa, Y., Hirano, T., Yoshimoto, K., Shimizu, M. \& Kobayashi, H. Non-invasive quantitative detection and applications of non-toxic, S65T-type green fluorescent protein in living plants. Plant J 18, 455-463 (1999).

39. Mueller, L. A., Goodman, C. D., Silady, R. A. \& Walbot, V. AN9, a petunia glutathione S-transferase required for anthocyanin sequestration, is a flavonoidbinding protein. Plant Physiol 123, 1561-1570 (2000).

40. Smith, A. P. et al. Arabidopsis AtGSTF2 is regulated by ethylene and auxin, and encodes a glutathione S-transferase that interacts with flavonoids. Plant J 36, 433-442 (2003)

41. Kumar, S. et al. Expression of a rice Lambda class of Glutathione S-transferase, OsGSTL2, in Arabidopsis provides tolerance to heavy metal and other abiotic stresses. Journal of Hazardous Materials 777, 228-237 (2013).

42. Olsen, A. N., Ernst, H. A., Leggio, L. L. \& Skriver, K. NAC transcription factors: structurally distinct, functionally diverse. Trends Plant Sci 10, 79-87 (2005).

43. Yasui, Y. et al. The phytochrome-interacting vascular plant one-zinc fingerl and VOZ2 redundantly regulate flowering in Arabidopsis. Plant Cell 24, 3248-3263 (2012).

44. McClung, C. R. Plant circadian rhythms. Plant Cell 18, 792-803 (2006).

45. Corbesier, L. et al. FT protein movement contributes to long-distance signaling in floral induction of Arabidopsis. Science 316, 1030-1033 (2007).

46. Lai, A. G. et al. CIRCADIAN CLOCK-ASSOCIATED 1 regulates ROS homeostasis and oxidative stress responses. Proc Natl Acad Sci U S A 109, 17129-17134 (2012).

47. Heidrich, K. et al. Arabidopsis EDS1 connects pathogen effector recognition to cell compartment-specific immune responses. Science 334, 1401-1404 (2011).

48. De-la-Pena, C. et al. Root secretion of defense-related proteins is developmentdependent and correlated with flowering time. J Biol Chem 285, 30654-30665 (2010).

49. Misra, P. et al. Modulation of transcriptome and metabolome of tobacco by Arabidopsis transcription factor, AtMYB12, leads to insect resistance. Plant Physiol 152, 2258-2268 (2010).

50. Pandey, A., Misra, P., Chandrashekar, K. \& Trivedi, P. K. Development of AtMYB12-expressing transgenic tobacco callus culture for production of rutin with biopesticidal potential. Plant Cell Rep 31, 1867-1876 (2012).

51. Seo, P. J. et al. A Golgi-localized MATE transporter mediates iron homoeostasis under osmotic stress in Arabidopsis. Biochem J 442, 551-561 (2012).

52. Felsenstein, J. An alternating least squares approach to inferring phylogenies from pairwise distances. Syst Biol 46, 101-111 (1997).

53. Choi, J. H., Jung, H. Y., Kim, H. S. \& Cho, H. G. PhyloDraw: a phylogenetic tree drawing system. Bioinformatics 16, 1056-1058 (2000).

54. Li, C. Automating dChip: toward reproducible sharing of microarray data analysis. BMC Bioinformatics 9, 231 (2008).

55. Clough, S. J. \& Bent, A. F. Floral dip: a simplified method for Agrobacterium mediated transformation of Arabidopsis thaliana. Plant J 16, 735-743 (1998)

56. Lee, Y. J., Kim, D. H., Kim, Y. W. \& Hwang, I. Identification of a signal that distinguishes between the chloroplast outer envelope membrane and the endomembrane system in vivo. Plant Cell 13, 2175-2190 (2001)

57. Tiwari, M. et al. Expression in Arabidopsis and cellular localization reveal involvement of rice NRAMP, OsNRAMP1, in arsenic transport and tolerance. Plant Cell Environ 37, 140-152 (2014).

58. Jefferson, R. A. The GUS reporter gene system. Nature 342, 837-838 (1989).

59. Yoo, S. D., Cho, Y. H. \& Sheen, J. Arabidopsis mesophyll protoplasts: a versatile cell system for transient gene expression analysis. Nat Protoc 2, 1565-1572 (2007).

\section{Acknowledgments}

This work was supported by a research grant from the Council of Scientific and Industrial Research, New Delhi, as Network Project (BSC-0107). Ashutosh Pandey, SRF (CSIR-NBRI) is kindly acknowledged for doing HPLC analysis. MT and DS thankfully acknowledge the Indian Council of Medical Research, India, and Council of Scientific and Industrial Research, India for Senior Research Fellowships.

\section{Author contributions}

M.T. and P.K.T. designed the experiments. M.T. and D.S. performed the experiments. M.T., R.D.T. and M.S. analyse the data and wrote the manuscript. All authors have read and approved the manuscript.

\section{Additional information}

Supplementary information accompanies this paper at http://www.nature.com/ scientificreports 
Competing financial interests: The authors declare no competing financial interests.

How to cite this article: Tiwari, M., Sharma, D., Singh, M., Tripathi, R.D. \& Trivedi, P.K. Expression of OsMATE1 and OsMATE2 alters development, stress responses and pathogen susceptibility in Arabidopsis. Sci. Rep. 4, 3964; DOI:10.1038/srep03964 (2014). (c) (i) $\odot$ This work is licensed under a Creative Commons AttributionBY No No Nommercial-NoDerivs 3.0 Unported license. To view a copy of this license, visit http://creativecommons.org/licenses/by-nc-nd/3.0 